\title{
A Modified Numerical Integration Method to Calculate the View Factor Between Finite and Infinite Cylinders in Arbitrary Array
}

\author{
Chuan Jiang ${ }^{\mathrm{a}, \mathrm{b}}$, Jun Wang ${ }^{\mathrm{a} *}$, Omar Behar ${ }^{\mathrm{b}, \mathrm{c}}$, Cyril Caliot ${ }^{\mathrm{b}}$, Yaoming Zhang ${ }^{\mathrm{a} *}$, Gilles \\ Flamant ${ }^{\mathrm{b}}$

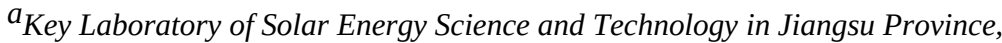 \\ Southeast University, School of Energy and Environment, No. 2 Si Pai Lou, Nanjing \\ 210096, China

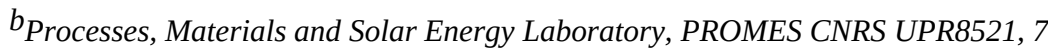 \\ Rue du Four Solaire, 66120 Font Romeu, France \\ ${ }^{C}$ Solar Energy Research Center (SERC-Chile), Av. Tupper 2007 Piso 4, Santiago, \\ Metropolitan Region, Chile
}

\begin{abstract}
:
The view factor has a critical impact on the estimation of the radiation heat transfer between different surfaces of energy systems. This paper presents an alternate and simple method for the blocking judgment and a modified numerical integration approach to calculate the view factor between finite and infinite length cylinders in an arbitrary array. The proposed method has been successfully validated through a comparison with the state-of-the-art published methods. Two cases are studied: (i) cylinders arranged in a staggered array for different spacing and lengths; (ii) cylinders arranged in an arbitrary array. The results show that the proposed method provides a fast and accurate way to evaluate the view factor for cylinder arrays with similar length. The analysis reveals that the length and arrangements have a strong influence on the view factor. The proposed method might be very useful to design an improved compact heat exchanger using a nonstandard arrangements.
\end{abstract}

Keywords: radiation heat transfer; view factor; numerical method; arbitrary cylinder array

\section{Introduction}

The geometry of a cylinder array is widely applied in industrial processes, i.e. heat pipe heat exchanger (HPHEX) in waste heat recovery system [1-3], rob bundles in nuclear reactor [4-6], pin-fin tubes [7-9], pin-fin heat sink for thermal management [10-18], solid oxide fuel cells (SOFCs) [19, 20] and solar receivers in concentrated solar power(CSP) system [21-23]. Many studies focused on the convective heat transfer between the fluid and the bundles' surface. However, in the case of high-emissivity or high-temperature surfaces, the two modes of heat transfer (convection and radiation) are of comparable magnitude [24]. Jung et al. [3] developed a numerical model to estimate the radiation effect of a high-temperature HPHEX. The results showed that the maximum relative error in the cold-side wall temperature is about $5.2 \%$, when the radiation heat transfer is not considered. The error increases with the increase in the wall temperature. Bopche et al. [4] investigated the contribution of radiative heat transfer in the total energy exchange of 18 rod bundle facility during LOCA (loss of coolant accident) situation, and found that $80 \%$ of heat energy is transferred by radiation heat transfer mode. Jang et al. [25] found that the radiation heat transfer represents about $25 \%$ of the total heat transfer rate in a pin-fin

* Corresponding author.

* E-mail address: 101010980@seu.edu.cn (Jun Wang) 101010988@seu.edu.cn (Yaoming Zhang) 
radial heat sink. These studies have revealed a non-negligible share of radiation heat transfer between cylinder arrays of different geometries. Thus, the evaluation of radiation heat transfer between cylinders is of particular interest.

Indeed, the evaluation of the radiation heat transfer within the cylinder array depends on the view factor or the geometric configuration factor [19]. The view factor is defined as the fraction of energy leaving one diffuse surface element (E1) and arrives at another element (E2) [26]. Many studies investigated the methods to get the view factor between the simplest case, i.e., view factor between two parallel cylinders. For the infinite long cylinders, an analytical expression can be derived by the crossed string method, which was first introduced by Hottel [27]. For the parallel cylinders of a finite length, Plamondon[28] developed a quadruple integral expression with limits of integration for the view factor between two parallel cylinders for a given radius ratio. Sparrow and Jonsson[29] investigated the view factor between two cylinders of finite length and equal radius by using contour integral technique. Juul[30] developed a new integration scheme, which can reduce the quadruple integral to a simple double integral. The simple double integral can only be carried out numerically. Besides, analytical expressions were developed, which approximate the view factor between two parallel cylinders of equal length and a given radius. The results showed that the error calculated by two methods was less than 1 percent.

In practice, cylinders are usually arranged in different arrangements. For example, the square arrangement[31], the circular arrangement or the hexagonal assembly[19, 20]. In these cases, the views between two parallel cylinders may be partially blocked by the adjacent cylinders. Therefore, it is difficult to get the analytical expression for the view factor except for the limiting case of infinite long cylinders by applying the crossed string method. Cox[32] derived the theoretical equations for view factors between infinite cylinders up to four rows on a triangular array and on a square array. The equations are expressed in a function of the ratio between the spacing and the diameter of the cylinder. Gerencser and Razani[33] proposed an approximate method to evaluate the view factor between one cylinder and all the other cylinders in a staggered array. For the cylinders of finite length in nonstandard arrangements, few studies focus on this problem. Bopche et al.[34] gave analytical expressions for the view factor between two finite length cylinders with interference from a third cylinder, by applying the contour integral technique. Yang et al.[35] investigated the view factor among rob bundles in 2-D with the nonstandard structure by applying the discrete transfer model (DTM) and discrete ordinates model(DOM) embedded in a CFD software. A direct numerical method is very useful to determine the view factor between two finite areas. But it requires careful consideration of the effects of shading or blocking by intervening surfaces, and the process can be quite computationally intensive in a complex system[26]. Some researchers proposed other methods to estimate the view factor in a complex, which could also be used in cylinder arrays, such as Monte Carlo Ray Tracing (MCRT) method[36, 37]. Many computer programs are available and use these methods, such as FLUENT, COMSOL, TracePro, FIDAP, NEVADA and so on. These methods and programs work well with complex geometries, but it is time-consuming and inefficient. For engineering applications, a fast and precious method is more suitable.

In this paper, a simple method for blocking judgment is proposed by applying the geometric analysis. Besides, a modified integration scheme is developed by using an analytical expression to calculate the view factor between two elemental strips, which could reduce the quadruple integral to a simple double integral. The next section describes the direct numerical integration method and 
proposes a modified numerical integration method to calculate the view factor. The the mesh sensitivity is analyzed in Section 3.1; the validation of the proposed method is presented in section 3.2; the effect of spacing and length on the view factor for different arrangements are discussed in Sections 3.3 and 3.4. Section 4 summarizes the most important conclusions.

\begin{tabular}{|c|c|}
\hline \multicolumn{2}{|l|}{ Nomenclature } \\
\hline$A$ & $\begin{array}{l}\text { Finite area of the surface }\left[\mathrm{m}^{2} i \text { or dimensionless parameter defined in Eq. (11), }\right. \\
(H / R)^{2}-\left(R_{0} / R\right)^{2}+1\end{array}$ \\
\hline$B$ & Dimensionless parameter defined in Eq.(11), $(H / R)^{2}+\left(R_{0} / R\right)^{2}-1$ \\
\hline C & Dimensionless parameter defined in Eq.(7), $H / l_{i j}$ \\
\hline$C_{i}$ & Center point of a cylinder/circle \\
\hline$D$ & Diameter of a circle or cylinder [m] \\
\hline$F_{1-i}$ & View factor from surface 1 to surface $i$ \\
\hline$F_{d A_{i}-d A_{j}}$ & View factor from elemental area $d A_{i}$ to the elemental area $d A_{j}$ \\
\hline$H$ & Length of a cylinder [m] \\
\hline$l_{i}$ & Projecting distance of a segment on a fixed direction [m] \\
\hline$l_{i j}$ & Distance between two elemental surfaces $[\mathrm{m} i$ \\
\hline$\vec{n}$ & Unit normal vector of an elemental surface \\
\hline$N$ & Number of elements \\
\hline$N_{d \theta}$ & Number of elements along the circumference \\
\hline$N_{d H}$ & Number of elements along the length \\
\hline$N_{\text {ray }}$ & Ray number \\
\hline$P$ & Spacing of two cylinders' center points [m] \\
\hline$P_{i}$ & Center point of an elemental surface \\
\hline$\widehat{P}_{i}{ }_{j}$ & Vector between the center points of two elemental surfaces \\
\hline$r$ & Radius of a circle or a cylinder [m] \\
\hline$R$ & Radius of a circle or a cylinder [m] \\
\hline$S$ & Distance from a center point to a common tangent line/plane [m] \\
\hline$S$ & Separation distance between two cylinders [m] \\
\hline$X$ & Coordinate on X-axis \\
\hline$Y$ & Coordinate on Y-axis \\
\hline$Z$ & Distance from a center point to a line $[\mathrm{m}]$ \\
\hline \multicolumn{2}{|c|}{ Greek symbols } \\
\hline$\alpha$ & Angle corresponding to an arc or segment $[\mathrm{rad} i$ \\
\hline$\beta$ & Angle corresponding to an arc or segment $[\mathrm{rad} i$ \\
\hline$\gamma$ & $\begin{array}{l}\text { Ratio of the view factor between two finite length cylinders to the view factor } \\
\text { between two infinite length cylinders }\end{array}$ \\
\hline$\theta$ & $\begin{array}{l}\text { Angle between the surface normal vector and the line between the center } \\
\text { points of two element surface }[\mathrm{rad} i\end{array}$ \\
\hline
\end{tabular}




$\xi \quad$ Blocking factor

\section{Method}

\subsection{Direct numerical integration method}

Fig.1 shows two parallel cylinders with the same length. In direct numerical integration method, the cylindrical surfaces are discrete into $N_{d \alpha} \times N_{d h}$ elements on length and on circumference. The view factor between the elemental area $d A_{i}$ on surface 1 and the elemental area $d A_{j}$ on surface 2 is given by:

$F_{d A_{i}-d A_{j}}=\frac{\cos \theta_{1} \cos \theta_{2}}{\pi l^{2}} d A_{j}$

Where: $l$ is the length of the line joining two areas and $\theta_{1}\left(\operatorname{or} \theta_{2}\right)$ is the angle between the surface normal vector and the line connecting two elemental areas.

Then the total view factor between two surfaces is given by:

$$
F_{1-2}=\frac{1}{A_{1}}\left\{\sum_{i=1}^{N_{d \mathrm{dc}} \times N_{d h}}\left(d A_{i} \sum_{j=1}^{N_{d \beta} \times N_{d h}} \xi F_{d A_{i}-d A_{j}}\right)\right\}
$$

Where: $\xi$ represents the blocking factor. It is usually obtained by calculating the intersection of the line between $d A_{i}$ and $d A_{j}$ and the surface, which may block the view. If the intersection exists, the view between two elementary areas is blocked by the surface and $\xi$ equals to 0 , if not, two elemental areas $d A_{i}$ and $d A_{j}$ can view each other and $\xi$ equals to 1.

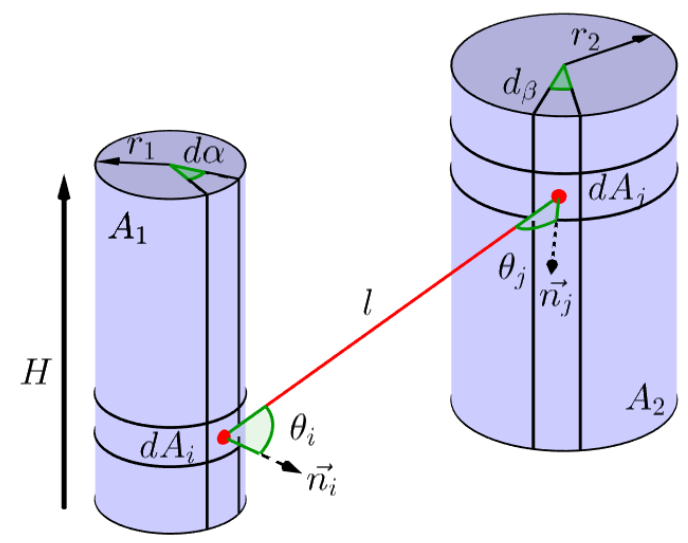

Fig.1. Basic geometry for view factor analysis

\subsection{Modified numerical integration method}

The cylindrical surface is formed by sweeping a circle along the Z-axis, so the elements along the Z-axis may be blocked by another cylinder surface at the same time. In this way, a novel blocking judgment was proposed, which is solved in 2-D. The detailed method is described below.

An arbitrary geometry of cylinder array with the same finite length and different radius is shown in Fig.2(a). The main purpose is to obtain the view factor between cylinders 1 and 2. Cylinder 3-5 are adjacent cylinders. Firstly, we need to select the adjacent cylinders, which may block the 
radiation from cylinder 1 to cylinder 2. As shown in Fig.2(b), the two common tangent lines/planes divide the cylindrical surface into two arcs. The arc facing outward in each cylinder can't see each other, which means the view factor between them equals to zero. The arcs facing inward consist of the area where radiation could happen with two common tangents. In order to reduce the calculation, the potential blocking cylinder should be selected by detecting if the circle and the polygon have an intersection or not. The approach consists of calculating the distances between the center point of the surrounding cylinders and two common tangents. If one of them is smaller than its radius, the cylinder is chosen for the calculation. Otherwise, which means both of them are larger than its radius, the position of the center point is detected. If the center point is inside the polygon (area of red dash line), such as circle/cylinder 4 in Fig.2(b), then it is also chosen for the calculation. Two common approaches (ray casting and angle summation) can be used to solve this problem [38].

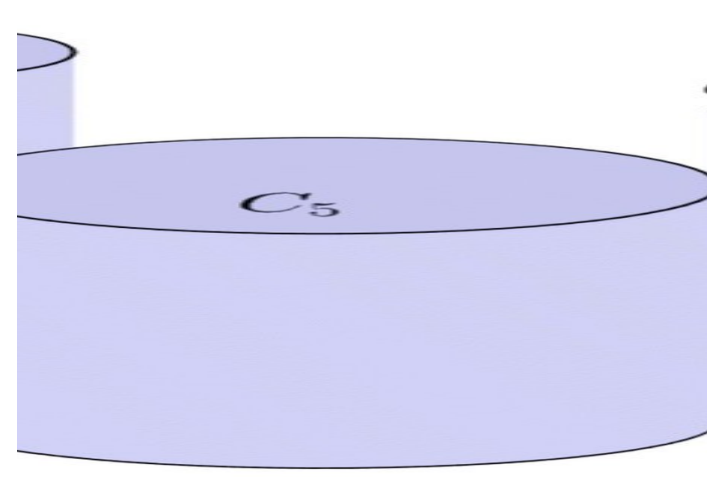

(a)

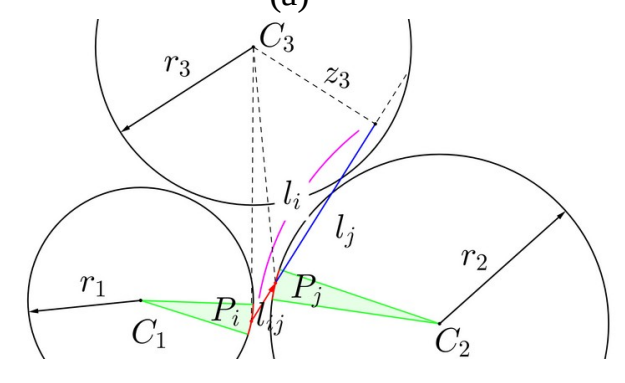

(c)

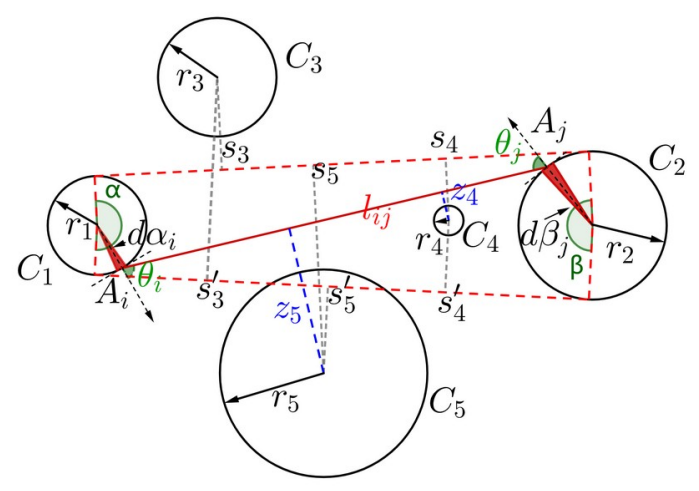

(b)

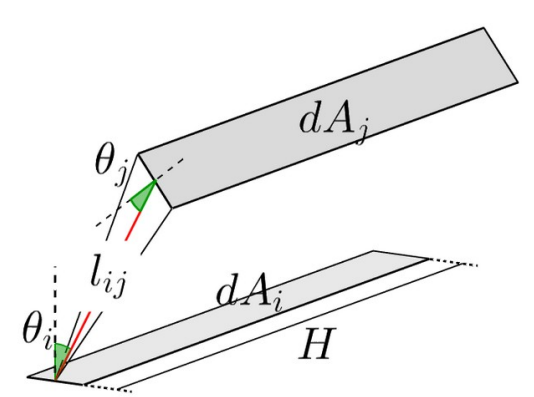

(d)

Figure.2 Geometry for view factor analysis: (a) Overview of finite cylinders in an arbitrary array; (b) Top view; (c) Special case of blocking judgment; (d) Two parallel elemental strips in the arbitrary configuration.

Then, the angle $\alpha$ corresponding to the arc facing inside is divided into $N_{d \alpha}$ parts and the cylinder is divided into $N_{d \alpha}$ strip elements respectively. $\alpha$ and $\beta$ can be calculated by:

$\alpha=2 \cos ^{-1} \frac{R-r}{P}, \beta=2 \pi-\alpha, R \geq r$

For any element $A_{i}$ in cylinder 1 and $A_{j}$ in cylinder 2, a second judgement is conducted to check if they can see each other without any interference. The mathematical description is:

$\theta_{i}<90^{\circ} \wedge \theta_{j}<90^{\circ}$ 
Or

$\vec{n}_{i} \cdot{\overrightarrow{P_{i} P_{j}}}_{j}>\wedge \vec{n}_{j} \cdot{\overrightarrow{P_{j} P_{i}}}>0$

Where $P_{i}$ and $P_{j}$ are the center points of the elements, $\vec{n}_{i j}$ is the unit normal vector of the element, $\theta_{i}$ and $\theta_{j}$ are the angles between the normal line and the line connecting two center points $\overline{P_{i} P_{j}}$. Then, in order to determine whether the adjacent cylinder blocks the view of two elements or not, a simple judgment is carried out by detecting whether the circle and the line segment $\overline{P_{i} P_{j}}$ have an intersection or not. Initially, we calculate the distance $z_{k}$ from the center point of an adjacent cylinder $k(k=3,4,5 \ldots)$ to the line $\overline{P_{i} P_{j}}$. If $z_{k} \leq r_{k}$, the view might be blocked. Only considering this condition could cause an error when the spacing is too small. Shown in Fig.2(c), the condition $Z_{3} \leq r_{3}$ is acquired, but cylinder 3 doesn't block the view between element $A_{i}$ and $A_{j}$. It is observed that in this case, the projection point of the center of cylinder 3 always locates on the extended line of $\overline{P_{i} P_{j}}$, instead of the segment. So, we calculate the projecting distances of $\overline{P_{i} C_{3}}$ and $\overline{P_{j} C_{3}}$ on the $\overline{P_{i} P_{j}}$ direction, which are $l_{i}$ and $l_{j}$ respectively. If the blocking happens, $l_{i}$ and $l_{j}$ should be less than or equal to the distance between two elements $l_{i j}$. Thus, the complete conditions for blocking are: (a) ${ }_{z_{k}} \leq r_{k} ;(b) l_{i} \leq l_{i j} \wedge l_{j} \leq l_{i j}$.

The blocking factor between any two elements $A_{i}, A_{j}$, and a cylinder $k$ is given by:

$\xi=\left\{\begin{array}{c}\dot{i} 0, z_{k} \leq r_{k} \wedge l_{i} \leq l_{i j}, l_{j} \leq l_{i j} \\ \dot{i} 1, \text { otherwise }\end{array}\right.$

Then, the view factor between two elemental strips of the same length is derived based on Jakob's study [39]:

$F_{A_{i}-A_{j}}=\xi \frac{\cos \theta_{i} \cdot d \phi}{\pi} \tan ^{-1} C$

$d \phi=2 \tan ^{-1} \frac{R_{j} d \theta_{j} \cdot \cos \theta_{j}}{2 l_{i j}}, C=\frac{H}{l_{i j}}$

When the cylinder is infinite, Eq. (7) becomes:

$F_{d A_{i}-d A_{j}}=\xi \frac{\cos \theta_{i} \cdot d \phi}{2}$

(8)

The total view factor between cylinder 1 and 2 is given by:

$F_{1-2}=\frac{1}{A_{1}}\left\{\sum_{i=1}^{N_{d \theta}}\left(A_{i} \sum_{j=1}^{N_{d \theta}} F_{d A_{i}-d A_{j}}\right)\right\}$

The proposed method is developed using an in-house MATLAB ${ }^{\circledR}$ code that has been developed tbased on the above equations.

\section{Validation and results}

Typical geometry of cylinder array in a staggered arrangement is shown in Fig.3 (top view). All the cylinders have the same radius $D$ and length $H$. The distance between the centers of two adjacent cylinders is equal to $P$. By considering symmetry, the cylinders with the same distance to the center one should have the same view factor. 


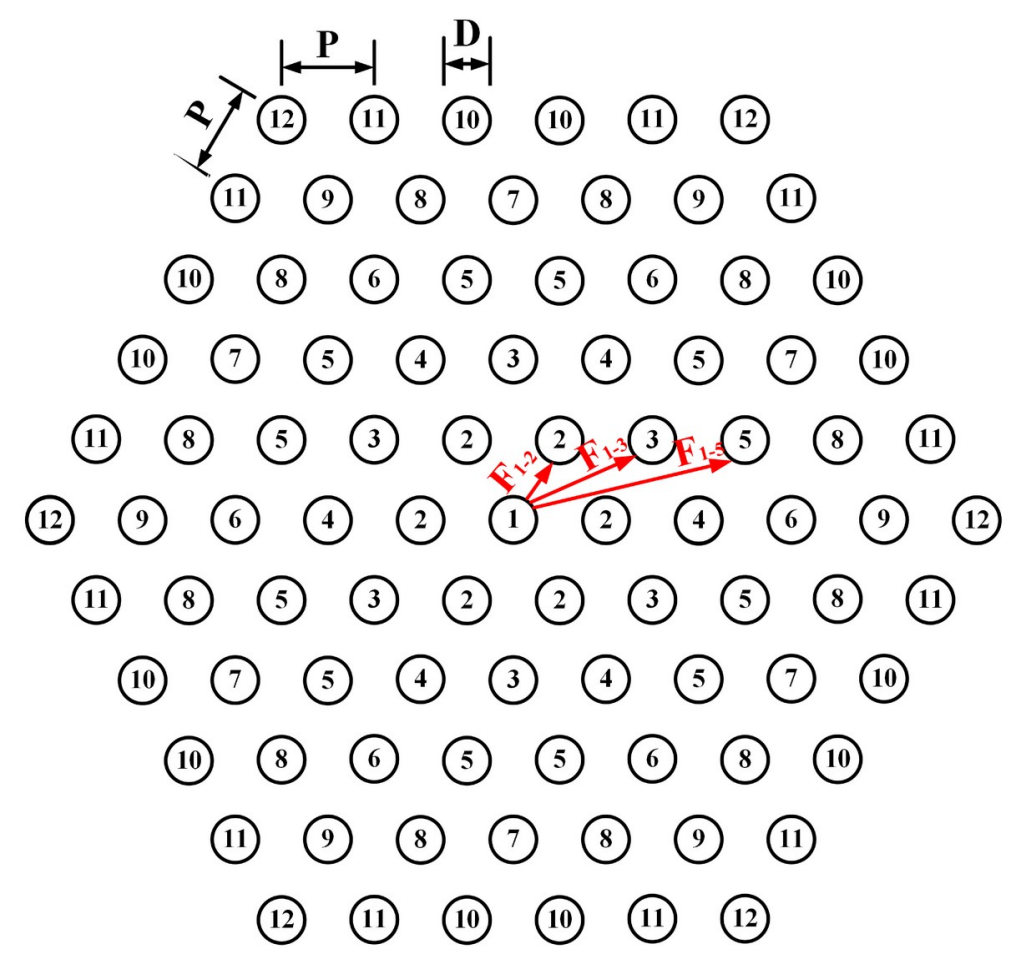

Fig.3. Cylinder arrays in a staggered arrangement (top view)

\subsection{Mesh sensitivity analysis}

The mesh sensitivity analysis should be made to verify the mesh number to meet the precision requirement. In the case shown in Fig.3, the ratio of pitch to diameter $P / D$ equals to 2 and the ratio of length to diameter $H / D$ equals to 1 . The total view factor between the center cylinder 1 to all the surround cylinder is calculated by the Mont Carlo Ray Tracing (MCRT) method[40], the direct numerical integration method and the method proposed in this study. The MCRT method evaluates the view factor by:

$F_{i, j}=\frac{N_{j}}{N_{i}}$

Where: $N_{i}$ is the total number of rays emitted from surface $i$ and $N_{j}$ is the total number of rays arrived on surface $j$.

In this paper, the MCRT method is conducted by software TracePro[41, 42]. Fig.4 and Fig.5 show the results of total view factor and computing time with different $N_{d \theta}$ and $N_{d h}$. The ray number $N_{\text {ray }}$ simulated in MCRT method ranges from $10^{4}$ to $10^{7}$. As illustrated, all the total view factors by different methods will converge to a value with the increasing $N_{d \theta}$ or $N_{\text {ray. }} . N_{d \theta}$ ranges from 10 to 1000 for both two numerical methods, and $N_{d H}$ equals to 5, 10, 20 and 30 only for direct numerical method. When $N_{d \theta}$ exceeds 200, the relative error by direct numerical method and modified numerical method is less than $0.012 \%$. The total view factor by using the direct numerical method decreases and approaches the curve by the modified numerical method when increasing the division number on length. When $N_{d H}=30, N_{d \theta}>200$, the relative error between the total view factor calculated by two methods is less than $0.012 \%$. The view factor obtained by the MCRT method has a large error with small numbers of ray. When ${ }_{N_{\text {ray }}}>8 \times 10^{6}$, the relative 
error of total view factor by MCRT method and modified numerical method is less than $0.02 \%$. Fig.5 shows the consuming time for each method as a function of $N_{d \theta}$ (Intel Core i5-4570 CPU 3.20GHz). The consuming time is the exponential function of $N_{d \theta}$ and the computing time by proposed method is much less than that of the direct numerical method. As can be seen, the computing time by the direct numerical method is 200 times more than the time consumed by the modified numerical method.

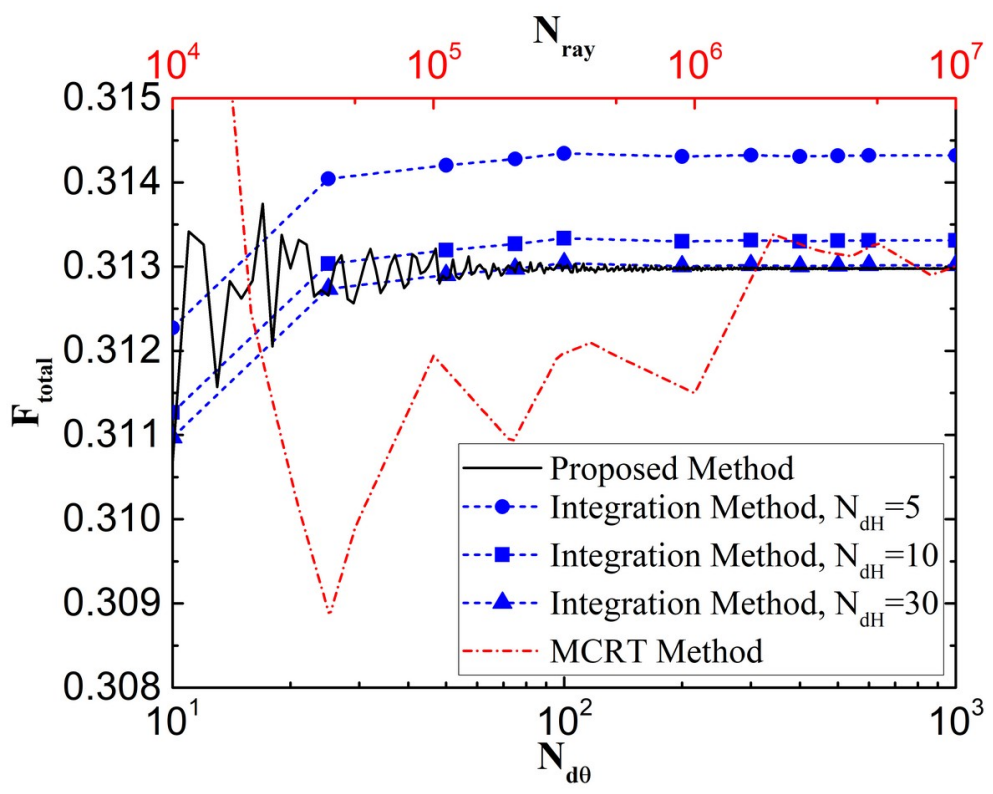

Fig.4 View factor between then center cylinder to the surrounding cylinders vs $N_{d \theta}$ and $N_{d H}$

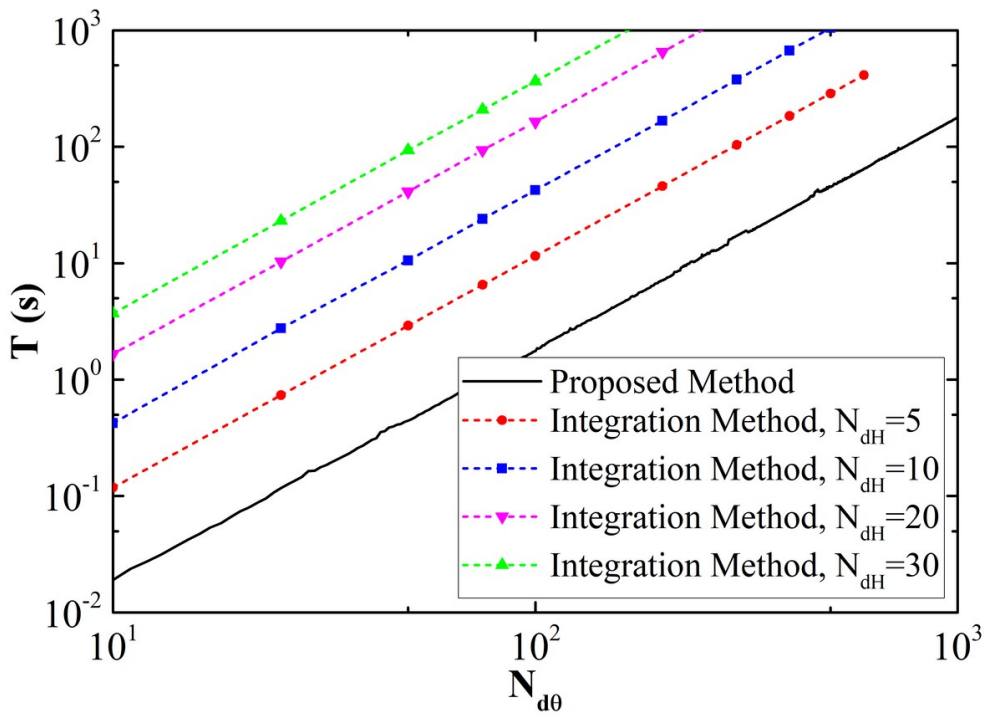

Fig.5 Computing time vs $N_{d \theta}$ and $N_{d H}$

\subsection{Validation}

Fig.6 compares the normalized view factor by the proposed method with the results of Juul [30]. The normalized view factor is defined as the ratio of the view factor for the case of a finite length to the view factor for the case of an infinite length. As shown in Fig.6, cylinders 1 and 2 are parallel with equal length $H$ but different radius $r$ and $R$ respectively. $S$ represents the separation distance. The view factors as a function of separation ratio $S / R$ for various length ratio $H / R$ and 
for two radius ratios $(r / R=1 \wedge 10)$ were calculated. The results showed that good agreements are achieved. The largest deviation of the two methods is less than $5 \%$.

In order to further verify the accuracy of the proposed method, the view factor between the center cylinder and the surrounding cylinders for the case of the geometry shown in Fig.3, for comparison with previously published methods. As illustrated in Table.1, the spacing/diameter equals to 2 and two cases are considered, i.e., the infinite length case and finite length case. For the infinite length case, the analytical expressions are derived by crossed string method[27, 32]. The complete expressions of view factor for 12 rows are given in Appendix A. Good agreement is obtained when comparing the proposed method and crossed string method. For the finite length case, because no published results found in the literature, the view factor was calculated by the MCRT method. The length/diameter equals to the unit and the ray number is 10 million. The results showed good agreement between the methods. The maximum relative error is $-3.4 \%$ for $F_{1-8}$.

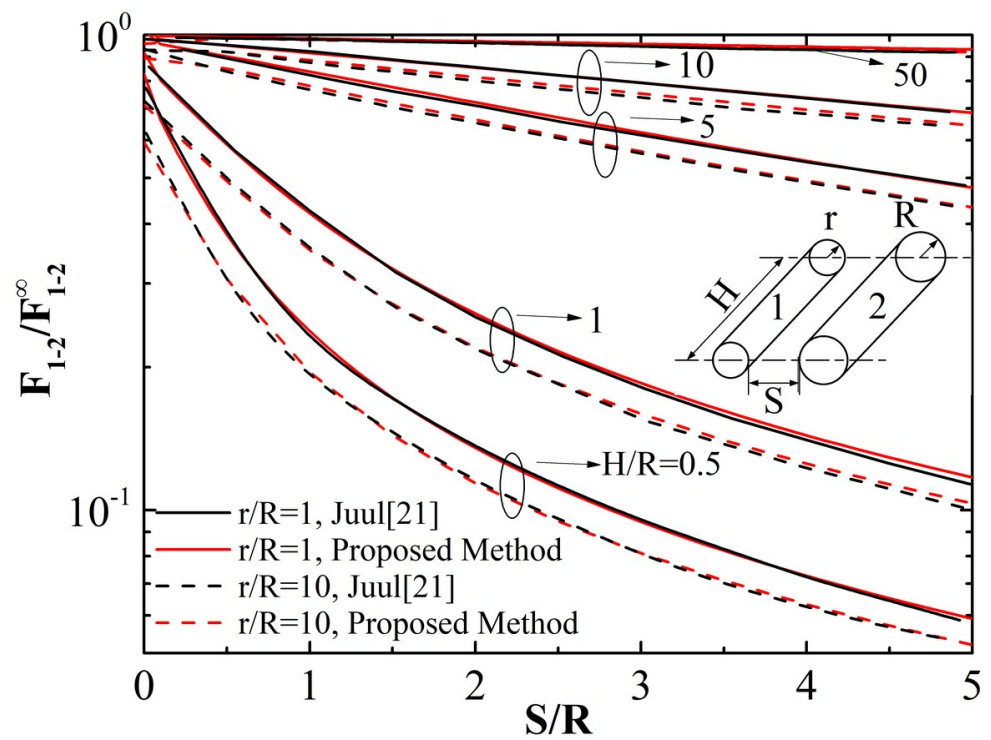

Fig.6 Comparison of normalized view factor between two parallel cylinders as a function separation ratio $S / R$ and various length ratios $H / R$ by proposed method and Juul[30]

Table. 1 comparison of view factors by different methods.

\begin{tabular}{lcccccc}
\hline & \multicolumn{5}{c}{ Spacing/diameter $(P / D)$, length/diameter $(H / D)$} \\
\cline { 2 - 6 } View & \multicolumn{3}{c}{$P / D=2, H / D=1$} & \multicolumn{3}{c}{$P / D=2, H / D=\infty$} \\
factor & $\begin{array}{c}\text { Proposed } \\
\text { method* }\end{array}$ & $\begin{array}{c}\text { MCRT } \\
\text { method } * *\end{array}$ & $\begin{array}{c}\text { Relative } \\
\text { error }\end{array}$ & $\begin{array}{c}\text { Proposed } \\
\text { method }\end{array}$ & $\begin{array}{c}\text { Crossed } \\
\text { string } \\
\text { method[32] }\end{array}$ & $\begin{array}{c}\text { Relative } \\
\text { error }\end{array}$ \\
\cline { 2 - 7 }$F_{1-2}$ & 0.03677 & 0.03680 & $-0.0185 \%$ & 0.08138 & 0.08138 & 0 \\
$F_{1-3}$ & 0.01064 & 0.01058 & $0.567 \%$ & 0.04627 & 0.04627 & 0 \\
$F_{1-5}$ & 0.00202 & 0.00202 & 0 & 0.01424 & 0.01425 & $-0.07 \%$ \\
$F_{1-8}$ & 0.00028 & 0.00029 & $-3.4 \%$ & 0.00290 & 0.00290 & 0 \\
\hline$* N_{d \theta}=200$ & & & & & \\
$* *$ & & & & &
\end{tabular}




\subsection{Effect of spacing and length on view factor for standard arrays}

The view factor $F_{1-i}(i=2,3,5,8,10,11)$ is plotted in Fig.7 (red-dash line) as a function of spacing ratio $P / D$ for various values of length ratio $H / D$. Considering the staggered array is one of the widely used arrangements in industrial applications, a simple analytical expression is more practical for calculation. In Ref[30], the ratio $y$ was introduced to estimate the ratio of the view factor between two finite length cylinders to the view factor between two infinite length cylinders. For the case of two cylinders with the same radius, the expression is given by the equation below:

$y=1-\frac{1}{\pi}\left\{\cos ^{-1} \frac{A}{B}-\frac{R}{2 H}\left[\sqrt{\left(A+2\left(R_{0} / R\right)^{2}\right)^{2}-\left(2 R_{0} / R\right)^{2}} \cos ^{-1}\left(\frac{R A}{R_{0} B}\right)+A \sin ^{-1}\left(\frac{R}{R_{0}}\right)-\frac{\pi}{2} B\right]\right\}$

$$
A=(H / R)^{2}-\left(\frac{R_{0}}{R}\right)^{2}+1^{\prime} B=(H / R)^{2}+\left(\frac{R_{0}}{R}\right)^{2}-1^{\prime} R_{0}=R \sqrt{\frac{2 \sqrt{(P / R)^{2}-1}-\pi}{2 \sin ^{-1}\left(\frac{R}{P}\right)}+1}
$$

The approximation is good when the blocking of the radiation from cylinder 1 to cylinder 2 by the adjacent cylinders is small. When $H / D=\infty, \gamma$ equals to unit. Combining the analytical expressions for infinite length cylinders in Appendix A, the view factor for finite length cylinders could be obtained by the equation below:

$F_{1-i}^{\text {finite }}=\gamma F_{1-i}^{\text {infinite }}$

The results calculated by equation (12) were also shown in Fig.7 (blue solid line). The relative error of the two methods is illustrated in Fig.8 by given a definition in eq. (13)

Relative Error $=\frac{\text { Proposed Method }- \text { Approximate Method }}{\text { Approximate Method }} \times 100 \%$

The view factor between the center cylinder and the nearest cylinder 2 was given in Fig.7 (a). $F_{1-2}$ decreases with the spacing rapidly and then tends to be gentle. According to the geometric analysis, when $P / D>2 \sqrt{3} / 3$, there is no blocking from cylinders 1 to cylinder 2 caused by the adjacent cylinders, which indicates that the view factor for two adjacent cylinders strongly depends on the spacing rather than on the blocking. Shown in Fig.8(a), Great agreement is achieved by two methods for various lengths when $P / D>1$, that the relative error is less than $0.6 \%$. It should be noticed that, when $P / D=1$, the view factor obtained by the proposed modified integration method has a large difference comparing with the results obtained by the approximate method. The maximum relative error is about $6.5 \%$ when $H / D=1$.The difference is mainly caused by using eq. (7) when they have common boundaries.

The cylinder length also has a significant effect on the view factor. As shown in Fig.7(a), when $P / D=2$, the view factor increases from 0.0367 to 0.0801 when $H / D$ increases from 1 to 50 . After that, the effect of length tends to be small. The relative error of the view factors when $H / D=50$ and $H / D=\infty$ is about $1.5 \%$ for $P / D=2$ and $6.6 \%$ for $P / D=6$. It is also found that the relative error between two methods decreases with the length.

Figs.7(b)-(f) illustrate the view factor from $F_{1-3}$ to $F_{1-11}$ as a function of spacing and length, and Figs.8(b)-(f) illustrate the relative error respectively. Generally, the view factor increases with the spacing in the beginning, reaches a peak value and decreases gradually. This is because of the 
interaction between the spacing and the blocking. When spacing increases, the effect of blocking by the former cylinders or adjacent cylinders becomes low. On the other hand, the view factor decreases with spacing. As can be seen in Fig.7(d), the curves might be divided into three regions. In region I, the objective surface (cylinder 8) is completely blocked by the cylinders in the front row, thus the view factor equals to zero. In region II, before the view factor reaches the highest value, the effect is dominated by blocking. After that, spacing has more effect than blocking. When the spacing is large enough, there is no blocking between cylinder 1 and cylinder 8 . For the standard arrangement, the spacing values for the three parts can be obtained by geometric analysis. The value of $P / D$ between the region I and II equal $2 \sqrt{3} / 3$. The value between region II and III equals to $2 \sqrt{39} / 3$. More details are given in Appendix A.

Figs.8(b)-(f) show the relative error of the view factors for the two methods. For all the view factors, the error decreases with the spacing ratio and length. With a large spacing ratio, like $P / D>4.5$, there is no difference between the two methods. On the other hand, the relative error for $F_{1-3}$ is relatively big when the spacing ratio is small. When $P / D$ is approaching 1 , the maximum error for $F_{1-3}$ could reach $34 \%$. However, considering the view factor starts from zero when $P / D=1$, The absolute error by the two methods is only $5.5 \times 10^{-5}(P / D=1.01, H / D=1)$, which could be neglected. The error decreases rapidly with the spacing ratio and length. Similarly, for view factor $F_{1-5}-F_{1-11}$, the maximum error is less than $20 \%$ and occurs at a small spacing value.

These results indicate that equations (11) and (12) are useful for both the case of two parallel cylinders without blocking as well as for the more complex case of finite length cylinders in an array. Using the approximate method is quite convenient, the only issue is the complex analytical expressions for infinite length case.

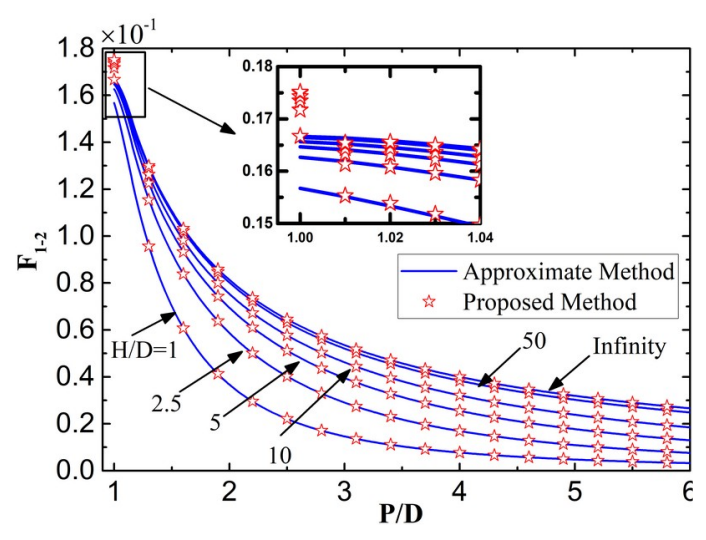

(a)

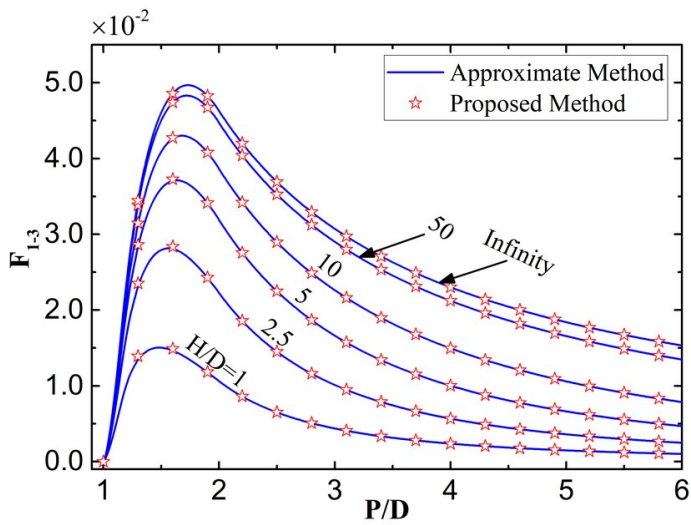

(b) 


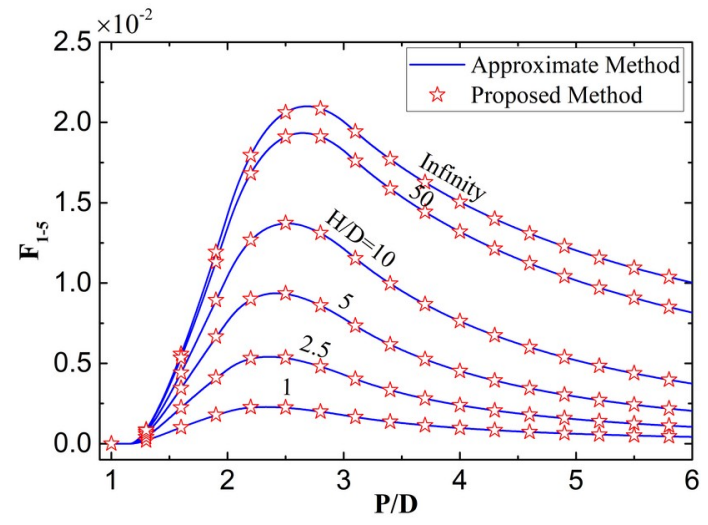

(c)

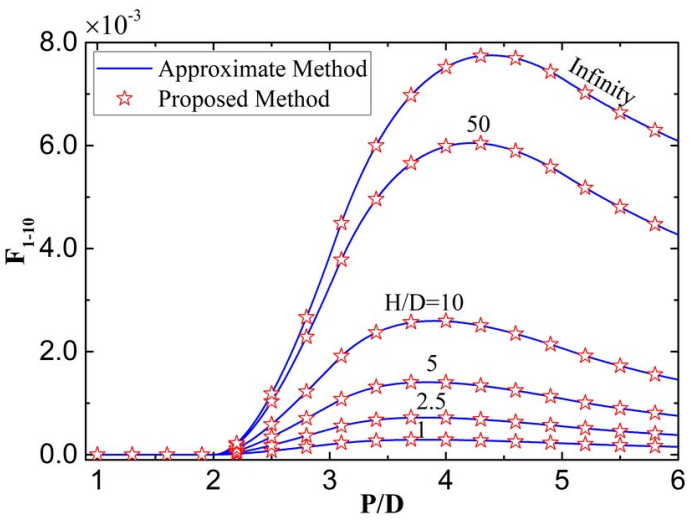

(e)

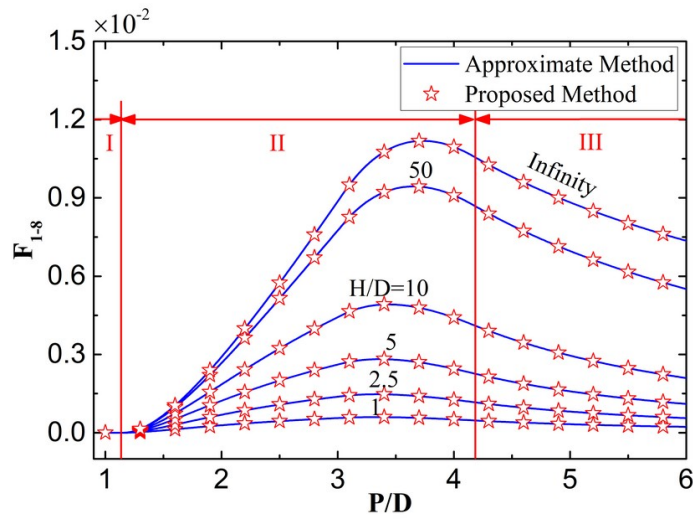

(d)

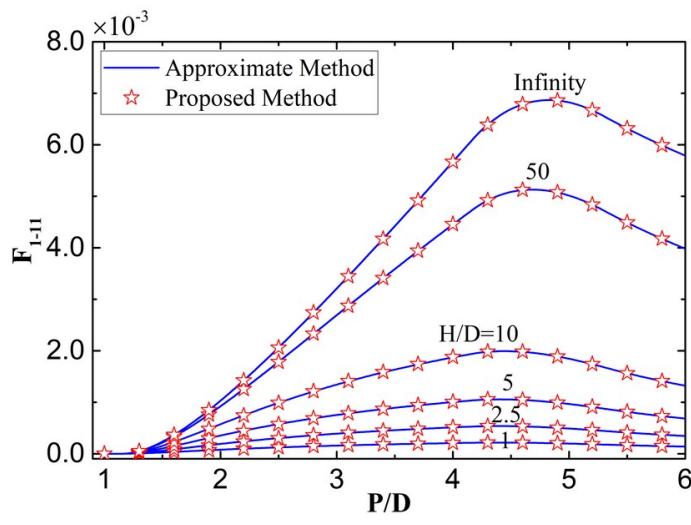

(f)

Fig.7 View factors vs spacing and length (a) $F_{1-2}$, (b) $F_{1-3}$, (c) $F_{1-5}$, (d) $F_{1-8}$, (e) $F_{1-10}$, (f) $F_{1-11}$.

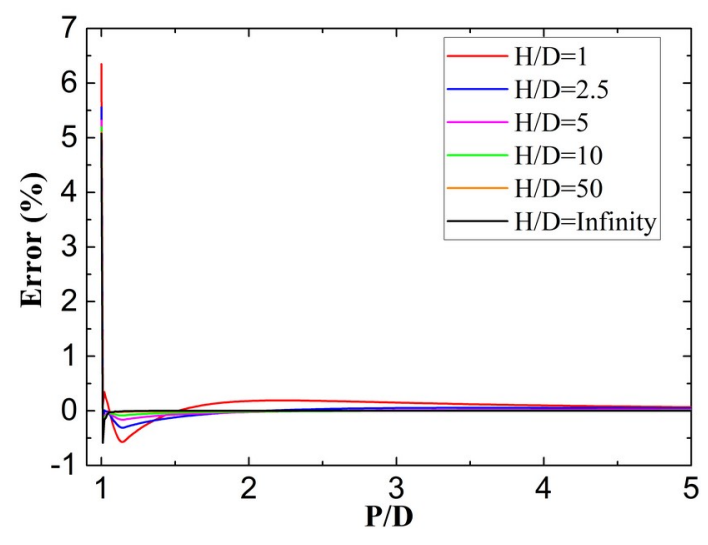

(a)

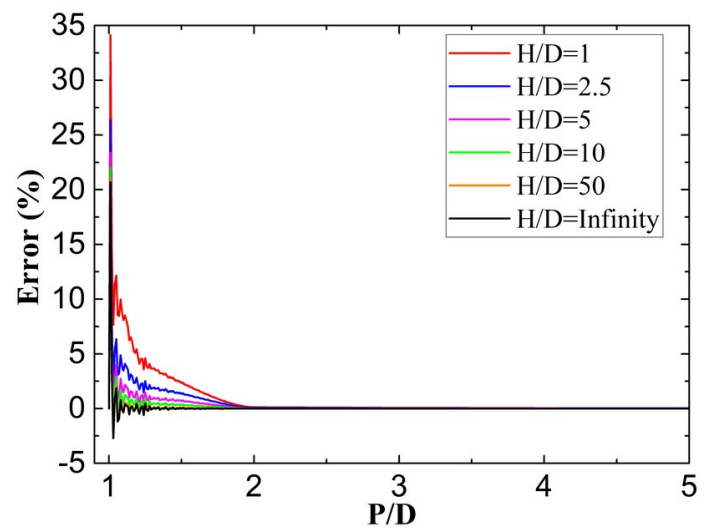

(b) 


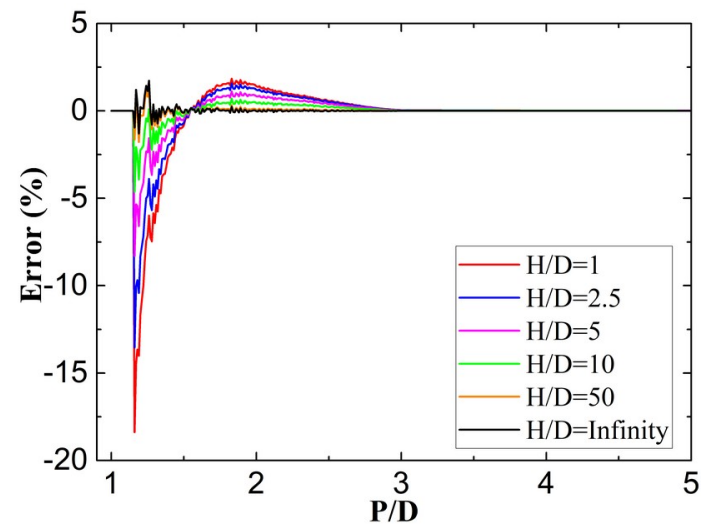

(c)

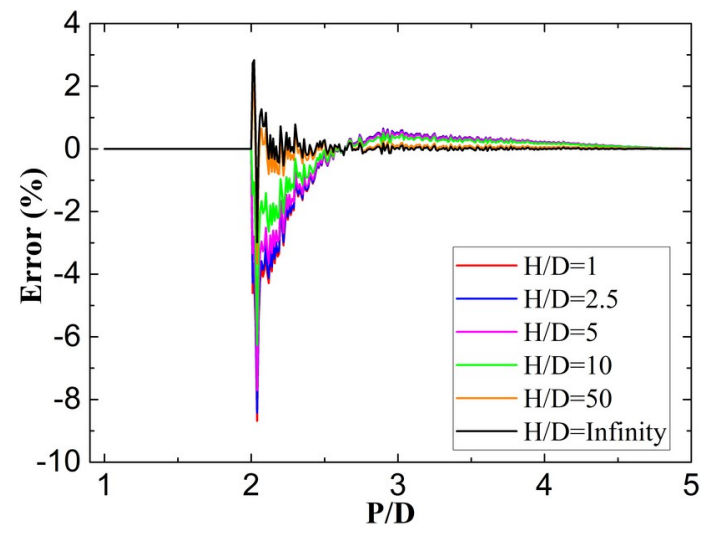

(e)

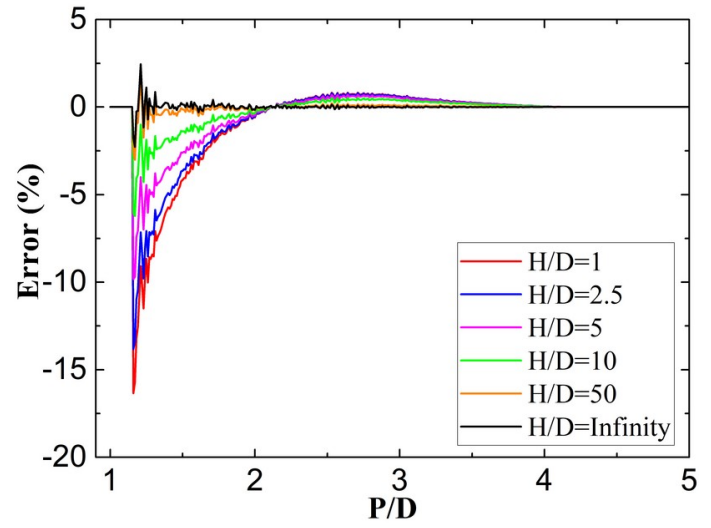

(d)

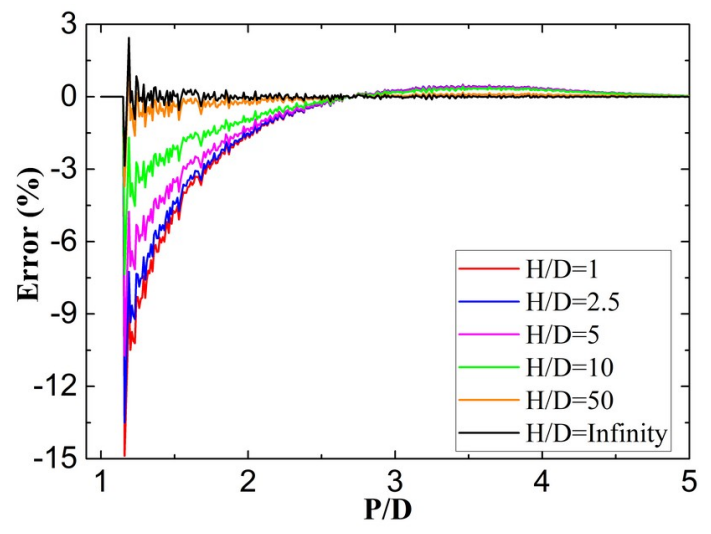

(f)

Fig.8 Relative error of view factors for modified numerical method and approximate method:
(a) $F_{1-2}$,
(b) $F_{1-3}$,
(c) $F_{1-5}$,
(d) $F_{1-8}$,
(e) $F_{1-10}$, (f) $F_{1-11}$.

\subsection{View factor calculation for a nonstandard arrangement}

For cylinder array in a nonstandard arrangement, it is difficult to get the view factor from the analytical methods, such as the crossed string method. Thus, the approximate method by combining equations (11) and (12) is not appropriate in this case. In order to solve this issue, the modified numerical method has been applied. A nonstandard arrangement for cylinder array is shown in Fig.9. Table.2 gives the diameter and coordinates for each cylinder. The view factor between cylinder 1 and 2 is calculated by the proposed modified numerical method (the divide number for both cylinders is 200). The result was plotted in Fig.10 as a function of length. The view factors were also compared with the results calculated by the MCRT method (ray number equals to $10^{7}$ ). A good agreement was found between the MCRT and the proposed method. As the arrangement was settled down, the view factor only changes with the length. Shown in Fig.10, when $H / D_{1} \leq 100, F_{1-2}$ increases rapidly with length. After that, length has less effect on the view factor. The ratio between the view factors for $H / D_{1}=1$ and $H / D_{1}=1000$ is about $15.7 \%$, whereas the ratio between that for $H / D_{1}=100$ and $H / D_{1}=1000$ is about $97.7 \%$. 


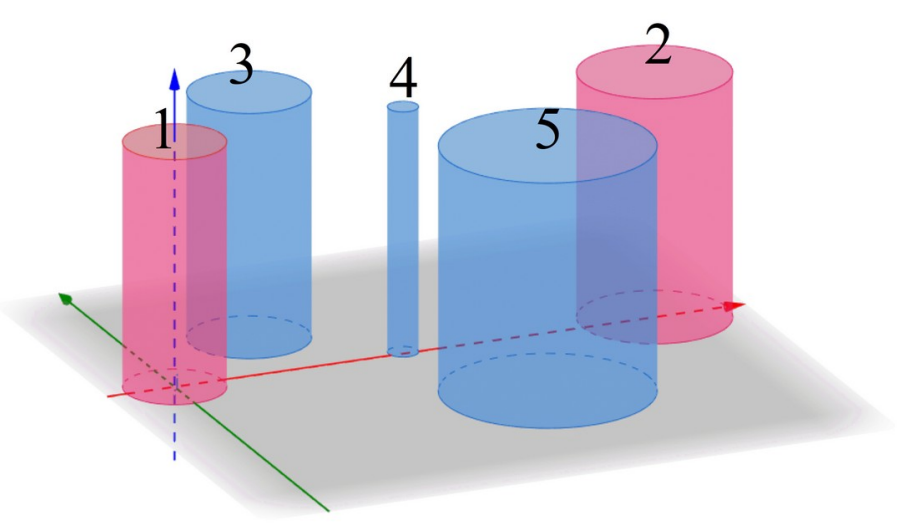

Fig.9 Geometry of nonstandard cylinder array.

Table.2 Parameters of nonstandard cylinder array

\begin{tabular}{cccccc}
\hline No. & 1 & 2 & 3 & 4 & 5 \\
\hline$D_{i}$ & 2 & 3 & 2.4 & 0.6 & 4.2 \\
$(X, Y)$ & $(0,0)$ & $(10,0)$ & $(2.4,2)$ & $(4.8,0.1)$ & $(6.5,-3)$ \\
\hline
\end{tabular}

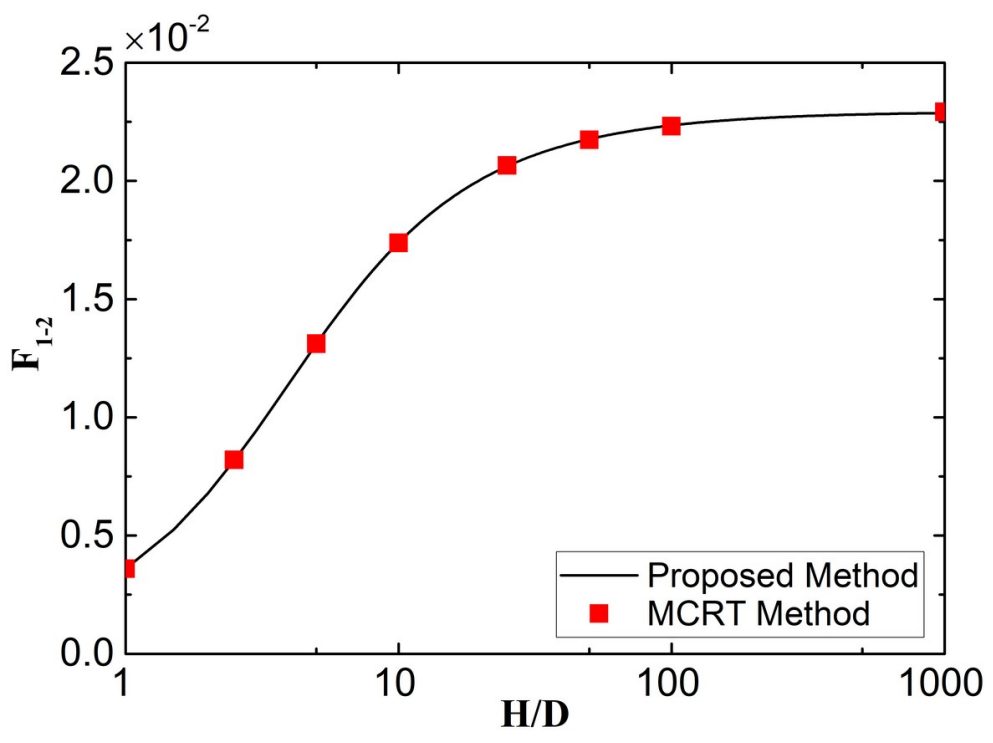

Fig.10 View factor between cylinders in nonstandard array

\section{Conclusion}

A modified numerical method to calculate the view factor between parallel cylinders of finite length in arbitrary arrangement is developed. The new method reduces the quadruple integration into a simple double numerical integration. Besides, a simple method to judge the blocking is also given. The novel method reduces the calculation time significantly. When compared with the direct numerical method it allows reducing $99.5 \%$ of the required time with similar accuracy. The method is applied to calculate the view factor between cylinder array in a staggered array. For the case of infinitely long cylinders, the result is compared with the crossed string method. For the case of finitely long cylinders, the result is compared with an approximated method. A good 
agreement has been found for all the cases. The analysis showed that the relative error decreases rapidly with the spacing ratio and length. The maximum relative error could reach $34 \%$ when cylinders are very close to each other. Besides, the spacing and the blocking affects the view factor between staggered cylinder arrays. Large spacing decreases the blocking but also the view factor. The maximum value exists except for the case without blocking. It has been observed that the view factor increases with the length, but the increasing tendency also declines with it. When $H / D>50$ for $1 \leq P / D \leq 6$, the relative difference between the finite length case and the infinite length case is less than $6.6 \%$. The results also indicate that the approximate method expressed by equation (11) and (12) has good agreement with the proposed modified numerical method. The precondition is to acquire the analytical expression for the infinite length case. For the case of cylinders in a nonstandard array, the proposed method gives accurate results as good as the MCRT method. In the case of a fixed arrangement, the view factor increases rapidly with the length in the beginning. After that, length has less effect on the view factor. The relative difference between the view factors for $H / D_{1}=100$ and $H / D_{1}=1000$ is only about $2.3 \%$. The proposed method might be very useful to design complex energy systems. For instance, it can be applied to design an improved compact heat exchanger in a nonstandard arrangement.

\section{Acknowledgments}

This work was supported by China Scholarship Council, French "Investments for the Future" ("Investissements d'Avenir") program managed by the National Agency for Research (ANR) under contract ANR-10-LABX-22-01 (labex SOLSTICE) and the National Science Foundation of China (No. 51736006).

\section{Appendix A:}

\begin{tabular}{|c|c|}
\hline \multicolumn{2}{|r|}{ View factor between infinite length cylinders in a staggered array } \\
\hline$F_{1-2}$ & $\begin{array}{l}F_{1-2}=\frac{1}{\pi}\left[\frac{\pi}{6}-\sqrt{(P / D)^{2}-1}+\cos ^{-1}\left(\frac{D}{P}\right)\right] \text { when } 1 \leq P / D \leq \frac{2 \sqrt{3}}{3} \\
F_{1-2}=\frac{1}{\pi} i\end{array}$ \\
\hline$F_{1-3}$ & $\begin{array}{l}F_{1-3}=\frac{1}{\pi} i \\
F_{1-3}=\frac{1}{\pi} i \\
F_{1-3}=\frac{1}{\pi}\left[\frac{\pi}{2}+\sqrt{(\sqrt{3} P / D)^{2}-1}-\sqrt{3} P / D-\cos ^{-1}\left(\frac{D}{\sqrt{3} P}\right)\right] \text { when } 2<P / D\end{array}$ \\
\hline$F_{1-5}$ & $\begin{array}{l}F_{1-5}=0 \text { when } 1 \leq P / D<\frac{2 \sqrt{3}}{3} \\
F_{1-5}=\frac{1}{2 \pi} i\end{array}$ \\
\hline
\end{tabular}




\begin{tabular}{|c|c|}
\hline & $\begin{array}{l}F_{1-5}=\frac{1}{\pi}\left[\sqrt{(\sqrt{7} P / D)^{2}-1}-\sqrt{(P / D)^{2}-1}-\sqrt{(\sqrt{3} P / D)^{2}-1}+\cos ^{-1}\left(\frac{D}{P}\right)+\cos ^{-1}\left(\frac{}{\sqrt{ }}\right.\right. \\
F_{1-5}=\frac{1}{\pi}\left[\frac{\pi}{2}+\sqrt{(\sqrt{7} P / D)^{2}-1}-\sqrt{7} P / D-\cos ^{-1}\left(\frac{D}{\sqrt{7} P}\right)\right] \text { when } \frac{2 \sqrt{21}}{3}<P / D\end{array}$ \\
\hline$F_{1-8}$ & $\begin{array}{l}F_{1-8}=0 \text { when } 1 \leq P / D<\frac{2 \sqrt{3}}{3} \\
F_{1-8}=\frac{1}{2 \pi}\left[\sqrt{(\sqrt{3} P / D)^{2}-1}+\sqrt{(\sqrt{13} P / D)^{2}-1}-2 \sqrt{(\sqrt{7} P / D)^{2}-1}+2 \cos ^{-1}\left(\frac{D}{\sqrt{7} P}\right.\right. \\
F_{1-8}=\frac{1}{\pi}\left[\sqrt{(\sqrt{13} P / D)^{2}-1}-\sqrt{(P / D)^{2}-1}-\sqrt{(\sqrt{7} P / D)^{2}-1}+\cos ^{-1}\left(\frac{D}{P}\right)+\cos ^{-1}\right. \\
F_{1-8}=\frac{1}{\pi}\left[\frac{\pi}{2}+\sqrt{(\sqrt{13} P / D)^{2}-1}-\sqrt{13} P / D-\cos ^{-1}\left(\frac{D}{\sqrt{13} P}\right)\right] \text { when } \frac{2 \sqrt{39}}{3}<P / D\end{array}$ \\
\hline$F_{1-10}$ & $\begin{array}{l}F_{1-10}=0 \text { when } 1 \leq P / D<2 \\
F_{1-10}=\frac{1}{2 \pi} i \\
F_{1-10}=\frac{1}{\pi}\left[\sqrt{(\sqrt{19} P / D)^{2}-1}-\sqrt{(\sqrt{7} P / D)^{2}-1}-\sqrt{(\sqrt{3} P / D)^{2}-1}+\cos ^{-1}\left(\frac{D}{\sqrt{3} P}\right)+c\right. \\
F_{1-10}=\frac{1}{\pi}\left[\frac{\pi}{2}+\sqrt{(\sqrt{19} P / D)^{2}-1}-\sqrt{19} P / D-\cos ^{-1}\left(\frac{D}{\sqrt{19} P}\right)\right] \text { when } 2 \sqrt{\frac{19}{3}}<P / D\end{array}$ \\
\hline$F_{1-11}$ & $\begin{array}{l}F_{1-11}=0 \text { when } 1 \leq P / D<\frac{2 \sqrt{3}}{3} \\
F_{1-11}=\frac{1}{2 \pi}\left[\sqrt{(\sqrt{7} P / D)^{2}-1}+\sqrt{(\sqrt{21} P / D)^{2}-1}-2 \sqrt{(\sqrt{13} P / D)^{2}-1}+2 \cos ^{-1}\left(\frac{1}{\sqrt{1}}\right.\right. \\
F_{1-11}=\frac{1}{\pi} i \\
F_{1-11}=\frac{1}{\pi}\left[\frac{\pi}{2}+\sqrt{(\sqrt{21} P / D)^{2}-1}-\sqrt{21} P / D-\cos ^{-1}\left(\frac{D}{\sqrt{21} P}\right)\right] \text { when } \frac{2 \sqrt{63}}{3}<P / D\end{array}$ \\
\hline
\end{tabular}

\section{References:}

[1]. Srimuang, W. and P. Amatachaya, A review of the applications of heat pipe heat exchangers for heat recovery. Renewable and Sustainable Energy Reviews, 2012. 16(6): p. 4303-4315.

[2]. Shabgard, H., et al., Heat pipe heat exchangers and heat sinks: opportunities, challenges, applications, analysis, and state of the art. International Journal of Heat and Mass Transfer, 2015. 89: p. 
138-158.

[3]. Jung, E.G. and J.H. Boo, Thermal numerical model of a high temperature heat pipe heat exchanger under radiation. Applied energy, 2014. 135: p. 586-596.

[4]. Bopche, S.B., A. Sridharan and A.K. Nayak, Evaluation of contribution of thermal radiation in transferring decay heat to the moderator, in case of 18 rod bundle facility, simulating advanced nuclear reactors. Progress in Nuclear Energy, 2015. 85: p. 83-95.

[5]. Senve, V. and G. Narasimham, Effective thermal conductivity of a heat generating rod bundle dissipating heat by natural convection and radiation. Nuclear Engineering and Design, 2011. 241(10): p. 4331-4340.

[6]. Xiong, J., et al., Experimental investigation on anisotropic turbulent flow in a $6 \times 6$ rod bundle with LDV. Nuclear Engineering and Design, 2014. 278: p. 333-343.

[7]. Ali, H.M. and A. Briggs, Condensation of R-113 on pin-fin tubes: effect of circumferential pin thickness and spacing. Heat Transfer Engineering, 2012. 33(3): p. 205-212.

[8]. Ali, H.M. and A. Briggs, Condensation of ethylene glycol on pin-fin tubes: Effect of circumferential pin spacing and thickness. Applied Thermal Engineering, 2012. 49: p. 9-13.

[9]. Ali, H.M. and A. Briggs, A semi-empirical model for free-convection condensation on horizontal pin-fin tubes. International Journal of Heat and Mass Transfer, 2015. 81: p. 157-166.

[10]. Micheli, L., K.S. Reddy and T.K. Mallick, Experimental comparison of micro-scaled plate-fins and pin-fins under natural convection. International Communications in Heat and Mass Transfer, 2016. 75: p. 59-66.

[11]. Ashraf, M.J., et al., Experimental passive electronics cooling: parametric investigation of pin-fin geometries and efficient phase change materials. International Journal of Heat and Mass Transfer, 2017. 115: p. 251-263.

[12]. Ali, H.M., et al., Thermal management of electronics: an experimental analysis of triangular, rectangular and circular pin-fin heat sinks for various PCMs. International Journal of Heat and Mass Transfer, 2018. 123: p. 272-284.

[13]. Wu, C., H. Tang and Y. Yang, Numerical simulation and optimization of turbulent flows through perforated circular pin fin heat sinks. Numerical Heat Transfer, Part A: Applications, 2017. 71(2): p. 172-188.

[14]. Kirsch, K.L. and K.A. Thole, Pressure loss and heat transfer performance for additively and conventionally manufactured pin fin arrays. International Journal of Heat and Mass Transfer, 2017. 108: p. 2502-2513.

[15]. Kong, D., et al., Single-phase thermal and hydraulic performance of embedded silicon micro-pin fin heat sinks using R245fa. International Journal of Heat and Mass Transfer, 2019. 141: p. 145-155.

[16]. Rehman, T., et al., A critical review on heat transfer augmentation of phase change materials embedded with porous materials/foams. International Journal of Heat and Mass Transfer, 2019. 135: p. 649-673.

[17]. Ali, H.M., et al., Preparation techniques of TiO2 nanofluids and challenges: a review. Applied Sciences, 2018. 8(4): p. 587.

[18]. Arshad, A., et al., An experimental study of enhanced heat sinks for thermal management using neicosane as phase change material. Applied Thermal Engineering, 2018. 132: p. 52-66.

[19]. Kee, R.J., B.L. Kee and J.L. Martin, Radiative and convective heat transport within tubular solidoxide fuel-cell stacks. Journal of Power Sources, 2010. 195(19): p. 6688-6698.

[20]. Hering, M., J. Brouwer and W. Winkler, Dynamic model of a micro-tubular solid oxide fuel cell 
stack including an integrated cooling system. Journal of Power Sources, 2017. 342: p. 504-514.

[21]. Wang, X., et al., Real - time thermal states monitoring of absorber tube for parabolic trough solar collector with non - uniform solar flux. International Journal of Energy Research, 2018. 42(2): p. 707719 .

[22]. Liao, Z. and A. Faghri, Thermal analysis of a heat pipe solar central receiver for concentrated solar power tower. Applied Thermal Engineering, 2016. 102: p. 952-960.

[23]. Bienert, W.B., The heat pipe and its application to solar receivers. Electric Power Systems Research, 1980. 3(1-2): p. 111-123.

[24]. Sparrow, E.M. and S.B. Vemuri, Orientation effects on natural convection/radiation heat transfer from pin-fin arrays. International journal of heat and mass transfer, 1986. 29(3): p. 359-368.

[25]. Jang, D., S. Yu and K. Lee, Multidisciplinary optimization of a pin-fin radial heat sink for LED lighting applications. International Journal of Heat and Mass Transfer, 2012. 55(4): p. 515 - 521.

[26]. Howell, J.R., M.P. Menguc and R. Siegel, Thermal radiation heat transfer. 2015: CRC press.

[27]. Hottel, H.C., Radiant heat transmission. WH McAdams. Heat Transmission, 1954.

[28]. Plamondon, J.A., Numerical determination of radiation configuration factors for some common geometrical situations. 1962: Jet Propulsion Laboratory, California Institute of Technology.

[29]. Sparrow, E.M. and V.K. Jonsson, Angle factors for radiant interchange between parallel-oriented tubes. Journal of Heat Transfer, 1963. 85(4): p. 382-384.

[30]. Juul, N.H., View factors in radiation between two parallel oriented cylinders of finite lengths. Journal of heat transfer, 1982. 104(2): p. 384-388.

[31]. George, N.M., et al., Neutronic analysis of candidate accident-tolerant cladding concepts in pressurized water reactors. Annals of Nuclear Energy, 2015. 75: p. 703-712.

[32]. Cox, R.L., Radiative heat transfer in arrays of parallel cylinders. 1976.

[33]. Gerencser, D.S. and A. Razani, Optimization of radiative-convective arrays of pin fins including mutual irradiation between fins. International journal of heat and mass transfer, 1995. 38(5): p. 899907.

[34]. Bopche, S.B. and A. Sridharan, Analytical expressions for configuration factor between cylindrical surfaces in rod bundle geometry. Nuclear Engineering and Design, 2010. 240(10): p. 30203036.

[35]. Yang, L., et al., Calculation of radiation heat transfer view factors among fuel rod bundles based on CFD method. Annals of Nuclear Energy, 2014. 71: p. 462-466.

[36]. Vujičić, M.R., N.P. Lavery and S. Brown, Numerical sensitivity and view factor calculation using the Monte Carlo method. Proceedings of the Institution of Mechanical Engineers, Part C: Journal of Mechanical Engineering Science, 2006. 220(5): p. 697-702.

[37]. Mirhosseini, M. and A. Saboonchi, View factor calculation using the Monte Carlo method for a 3D strip element to circular cylinder. International Communications in Heat and Mass Transfer, 2011. 38(6): p. 821-826.

[38]. Sutherland, I.E., R.F. Sproull and R.A. Schumacker, A characterization of ten hidden-surface algorithms. ACM Computing Surveys (CSUR), 1974. 6(1): p. 1-55.

[39]. Jakob, M., Heat Transfer. Vol. Volume. 1957, New York: John Wiley \& Sons.

[40]. Sparrow, E.M., Radiation heat transfer. 2018: Routledge.

[41]. https://www.lambdares.com/tracepro/.

[42]. Jafrancesco, D., et al., Strategy and criteria to optically design a solar concentration plant. Renewable and Sustainable Energy Reviews, 2016. 60: p. 1066-1073. 
